\title{
Essais
}

ESSAIS

Revue interdisciplinaire d'Humanités

Hors-série 4 | 2018

Stanley Kubrick

\section{Les Masques de la vanité. Kubrick, Schnitzler, Ophüls, Maupassant}

\section{Emmanuel Plasseraud}

\section{(2) OpenEdition}

1 Journals

Édition électronique

URL : http://journals.openedition.org/essais/685

DOI : 10.4000/essais.685

ISSN : 2276-0970

Éditeur

École doctorale Montaigne Humanités

\section{Édition imprimée}

Date de publication : 1 juillet 2018

Pagination : 97-104

ISBN : 979-10-97024-04-8

ISSN : 2417-4211

Référence électronique

Emmanuel Plasseraud, «Les Masques de la vanité. Kubrick, Schnitzler, Ophüls, Maupassant », Essais [En ligne], Hors-série 4 | 2018, mis en ligne le 01 décembre 2019, consulté le 16 décembre 2019. URL http://journals.openedition.org/essais/685; DOI : 10.4000/essais.685 


\section{Les Masques de la vanité. Kubrick, Schnitzler, Ophüls, Maupassant}

\section{Emmanuel Plasseraud}

Dernier film de Stanley Kubrick, Eyes Wide Shut (1999) est une adaptation d'une nouvelle d'Arthur Schnitzler, Traumnovelle, parue en $1925 .{ }^{1} \mathrm{Ce}$ récit, qui se déroule à Vienne, est transposé par Kubrick et son scénariste, Frederic Raphael, à New-York, à la fin du XX' siècle. Il raconte l'aventure d'un médecin (Bill Harford) qui, vexé par la révélation faite par sa femme (Alice) qu'elle a éprouvé du désir pour un autre homme, essaie de la tromper et se retrouve notamment dans une orgie dont il n'aurait jamais dû faire partie. Du fait du changement d'époque et de lieux, mais aussi de la difficulté à adapter au cinéma le texte de Schnitzler, qui est émaillé de réflexions intérieures, cette transposition ne pouvait que provoquer un certain nombre de changements importants par rapport au texte originel. Pourtant, comme Michel Chion l'a constaté en analysant le film, Kubrick en suit "la ligne narrative avec une étonnante fidélité $"^{2}$, prenant le risque que ce récit d'adultère fantasmé paraisse désuet à des spectateurs de l'an 2000. Il y a tout de même quelques libertés que Chion repère, comme l'ajout d'un personnage nouveau, Ziegler (incarné par Sidney Pollack). Les objets du monde de la fin du vingtième siècle interviennent parfois, comme lorsque Bill, qui s'apprête à avoir une relation avec une prostituée, est interrompu par un coup de téléphone de sa femme, alors que dans le livre, il renonçait de lui-même. Inversement, des aspects devenus trop lointains culturellement sont gommés dans le film, comme le phénomène de télépathie onirique qu'on trouve chez Schnitzler - la femme rêve que son mari se trouve dans une orgie qui tourne mal pour lui -, qui n’a pas été repris.

À propos du personnage de Ziegler, principale innovation par rapport à la Traumnovelle, Chion mentionne le témoignage de Jan Harlan, qui explique qu'il "provient d'une envie de Kubrick qu'un personnage de la vie quotidienne de

1 Arthur Schnitzler, "Rien qu'un rêve ", repris dans Les Dernière cartes, Paris, Calmann-Lévy, 1953, p. 125-220. La nouvelle est plus connue aujourd'hui sous le titre La Nouvelle rêvée.

2 Chion Michel, Stanley Kubrick, L’humain, ni plus ni moins, Paris, Cahiers du Cinéma, 2005, p. 452. 
Bill fasse le lien avec (la) société secrète $"^{3}$ qui organise l'orgie. Chion remarque d'autre part qu'il "appartient indubitablement à la famille des figures paternelles choquantes et libidineuses, provocantes, unissant intelligence et grivoiserie, que l'on retrouve dans toute l'auvre de Kubrick ${ }^{4}$. Mais ce personnage, ou plus exactement les modifications scénaristiques qu'engendre sa présence, possède deux autres fonctions qui pourraient expliquer aussi son invention.

La première de ces fonctions est de l'ordre de la stratégie scénaristique, permettant la mise en place en début de récit d'un implant narratif qui prépare la fin du film. En effet, c'est chez Ziegler que se déroule un événement important pour la suite du récit, qui n'apparait pas dans la nouvelle de Schnitzler : le malaise d'une prostituée, avec qui Ziegler a couché, à la suite d'une overdoses. Appelé à son chevet, Bill parvient à la ranimer, mais la prévient qu'elle doit faire une cure pour se sortir de son addiction, qui pourrait lui coûter la vie. Or, à la fin du film, c'est cette prostituée qui est retrouvée morte d'une overdose dans une chambre d'hôtel. Entretemps, elle a "sauvé " Bill durant l'orgie en le rachetant. Bill pense que derrière cette overdose se cache un meurtre commis par des membres de la société secrète, ce qu'il explique à la fin du film à Ziegler, dans une scène ajoutée par Kubrick et Raphael au récit de Schnitzler. Ziegler y révèle à Bill qu'il est au courant de son intrusion durant la cérémonie orgiaque puisqu'il y était aussi. Il tente de lui faire comprendre que les membres de la société secrète n'ont fait que chercher à l'effrayer, qu'ils n'ont donc pas le pouvoir qu'imagine Bill et qu'ils ne sont pas non plus responsables de la mort de la prostituée, dont le mode de vie était à risque. D'où l'intérêt de l'implant narratif de début de film, qui légitime l'opinion de Ziegler et fait douter Bill et le spectateur : ce que Bill pense être un meurtre n'est peut-être qu'un accident, qui d'ailleurs était prévisible étant donné l'hygiène de vie de la jeune femme. De cette manière, Kubrick substitue à la question posée à la fin de la Traumnovelle sur l'identité de la victime celle de l'implication de la société secrète dans sa mort, orientant le récit vers un questionnement sur la véracité de ce que Bill a vu. Réalité et faux-semblants se mêlent, dans une indistinction qui fait écho au thème principal du film, résumé dans le dialogue final entre Bill et sa femme : rêves et fantasmes font partie de la réalité au point que celle-ci ne peut plus être tout à fait distinguée d'eux.

L'autre fonction de l'ajout du personnage de Ziegler, ou plutôt de cette scène où une prostituée fait une overdose dans ses bras, est d'ordre intertextuel. En effet, l'intertextualité d'Eyes Wide Shut ne se limite pas à l'inter-

3 Ibid., p. 460.

4 Ibid., p. 460.

5 Il y a d'ailleurs un autre implant narratif, puisque le pianiste ami d'enfance de Bill Nightingale - important car il indique à Bill l'existence des cérémonies orgiaques - y apparait, alors que chez Schnitzler, le médecin le rencontre par hasard dans un café. 
textualité hétérofilmique propre à la pratique de l'adaptation. Il y a aussi, de manière moins flagrante, une intertextualité homofilmique sous la forme d'une allusion à un autre film ${ }^{6}$. Frederic Raphael a mentionné, comme sources d'inspiration, le Décalogue de Krzysztof Kieślowski ${ }^{7}$, Maris et femmes (1992) de Woody Allen, ou encore Pulp fiction (1994) de Quentin Tarantino (pour son tempo, ce qui est assez curieux étant donné la lenteur voulue d'Eyes Wide Shut, notamment dans la déclamation des dialogues) ${ }^{8}$. À sa sortie, le film a été rapproché d'After hours (1986) de Martin Scorsese, pour l'errance nocturne. Mais l'ajout de la séquence de l'overdose, durant la soirée de Ziegler, constitue aussi, et peut-être plus fondamentalement que tous ces rapprochements avec d'autres films, une allusion au film Le Plaisir de Max Ophüls (1952), et à son premier sketch, intitulé "Le Masque " ${ }^{9}$. Cette allusion a en tout cas le mérite de permettre d'éclairer une dimension importante du film de Kubrick.

À la fin de ce sketch, qui est adapté d'une nouvelle de Maupassant, un médecin venu raccompagner chez lui un vieil homme qui s'est écroulé de fatigue sur la piste de danse d'un bal, reçoit de la femme de ce noceur invétéré la leçon Ophülsienne par excellence : l'homme dans sa quête vaine du plaisir, rend la femme malheureuse en amour, elle qui aspire au bonheur conjugal ${ }^{10}$. Le sketch s'achève dans une rue montmartroise, alors que le docteur remonte dans sa calèche. Mais a-t-il retenu la leçon ? Le docteur songe qu'il vient de voir "une scène de l'éternel drame qui se joue tous les jours, sous toutes les formes, dans tous les mondes ", selon les termes de la voix off qui est censée être celle de Maupassant. Toutefois, cette phrase ne provient pas de la nouvelle elle-même, qui se clôt avant le retour du docteur au bal. Chez Ophüls en effet, contrairement à Maupassant, le docteur demande à son cocher de le ramener au Palais de la danse, tandis qu'on entend déjà une musique endiablée entendue auparavant durant le bal.

Ce docteur qui n'a donc pas retenu la leçon, que les plaisirs de la fête tentent plus que la sagesse, pourrait être un ancêtre de Bill Harford. Eyes Wide Shut apparaîtrait alors comme un prolongement et une variation sur le thème

6 L'intertextualité hétérofilmique désigne la référence d'un film à une œuvre qui n'est pas un autre film (le roman de Schnitzler ou encore l'utilisation du morceau Musica ricercata de György Ligeti dans Eyes Wide Shut) tandis que l'intertextualité homofilmique désigne un renvoie d'un film à un autre film. La notion d'intertextualité, d'origine littéraire, a été élaborée par Julia Kristeva dans Sémiotiké, recherches pour une sémanalyse, Paris, Seuil, 1969.

7 Sur l'influence du Décalogue 3 de Kieslowski sur Eyes Wide Shut, voir Gaspard Delon, "Échange de regards : Schnitzler, Kubrick et Kieslowski ", dans Cinéma, littérature : projections, études réunies et présentées par Marie Martin, La Licorne, $n^{\circ} 116$, Rennes, Presses Universitaires de Rennes, 2015, p. 187-199.

8 Chion Michel, Stanley Kubrick, L’humain, ni plus ni moins, op. cit., p. 460.

9 Par ailleurs, Ophüls a lui aussi adapté un roman de Schnitzler, La Ronde, en 1950.

10 Sur ce thème, voir Claude Beylie, Max Ophüls, Paris, Lherminier, Cinema classique - les cinéastes, 1984. 
Ophülsien - et plus généralement baroque, d'ailleurs - de la vanité. Mais d'un film à l'autre, d'une époque à l'autre, d'une référence littéraire à l'autre, la vanité change de forme et de sens, tout en s'accompagnant de figures récurrentes. Les situations dramatiques initiales sont différentes, tout en possédant une certaine similarité. Chez Ophüls, un médecin, qui profite d'un bal pour faire la rencontre de femmes - ce qui n'est d'ailleurs pas précisé chez Maupassant -, est appelé au chevet d'un danseur qui cache son âge avancé derrière un masque ${ }^{11}$. Dans Eyes Wide Shut, le médecin n'est plus seulement un témoin ; il devient le personnage central de l'intrigue. À la différence du médecin d'Ophüls, Bill Harford est marié, information donnée dès la première scène du film au spectateur. Il se rend avec sa femme à un bal, au cours duquel on lui demande d'intervenir pour une urgence médicale, alors qu'il flirte avec deux femmes. C'est alors qu'intervient la bifurcation entre l'intertextualité hétérofilmique et l'intertextualité homofilmique, car la scène où le médecin flirte avec deux femmes tandis que son épouse danse avec un inconnu étranger se trouve bien chez Schnitzler ${ }^{12}$. Mais elle s'arrête d'elle-même, alors que dans Eyes Wide Shut, c'est une urgence médicale qui l'interrompt. Or, c'est justement ce qui arrive au médecin d'Ophüls, alors qu'il se trouve lui aussi en charmante compagnie. Il découvre alors l'homme âgé qui se cache derrière son masque, qui a fait un malaise en compagnie, d'une danseuse, donc un couple ressemblant à celui formé par Ziegler et la call-girl (sauf que c'est la prostituée qui a fait un malaise chez Kubrick, et non l'homme âgé). De plus, le bal d'Eyes Wide Shut n'est pas masqué, comme chez Maupassant et Ophüls, alors qu'il l'est chez Schnitzler. Cela peut aussi se comprendre si l'on fait l'hypothèse qu'en adaptant Schnitzler, Kubrick et son scénariste ont songé au récit d'Ophüls-Maupassant, où le bal ne pouvait être masqué dans la mesure où il fallait que le vieux danseur masqué se distingue par cet attribut même des autres noceurs, plus jeunes que lui.

De cette bifurcation intertextuelle, qui semble mener au film d'Ophüls plus même qu'au récit de Maupassant, peut-on extrapoler et envisager une référence plus générale qu'en ce point précis ? Les deux scènes de bal sont très différentes : au rythme effréné de la musique populaire, des danseurs et de la caméra du film d'Ophüls, Kubrick a préféré un rythme lent : une

11 Ophüls insiste plus sur ce médecin que Maupassant, qui se concentre sur le couple du danseur âgé et de sa femme. En effet, de nombreux dialogues où la femme explique quelle a été sa vie avec son mari noceur ont été coupés dans le film d'Ophüls, qui en revanche comprend des scènes inédites destinées à mieux caractériser le médecin (dialogue avec une femme lors du bal, aveu qu'il est célibataire et qu'il fréquente les bals, morale de l'histoire et retour au bal). Tandis que Maupassant concentre son portrait sur le danseur âgé dont il fait, à travers son masque, un symbole de la vanité de la lutte contre le vieillissement, Ophüls fait de ce personnage le reflet de ce qu'est en train de devenir le médecin, qui semble en prendre conscience sans toutefois changer son comportement.

12 L'inconnu est polonais chez Schnitzler, hongrois chez Kubrick. 
danse langoureuse, au son d'une musique classique, des paroles échangées avec retenue, chuchotées, des mouvements de caméra planants réalisés au steadycam. De ce point de vue, le bal d'Eyes Wide Shut s'oppose à celui du Plaisir. Ce n'est donc pas sur ce plan qu'il faut chercher une forme d'allusion. Kubrick n'a pas non plus conservé les figures accompagnant le thème de la vanité chez Ophüls : la chute (le danseur âgé s'écroule sur la piste) et les ombres, symboles de l'inconsistance (le plan sur le visage masqué du danseur âgé, sur lequel s'agitent les ombres des danseurs). Il a choisi un objet symbolique qui revient souvent dans le film, sans d'ailleurs être mentionné dans le texte de Schnitzler : le miroir, devant lequel aussi bien Bill, seul, qu'Alice, seule aussi, ou les deux ensembles se regardent. En revanche, Le Plaisir et Eyes Wide Shut ont en commun deux autres figures de la vanité : le masque, même si celui-ci n'a pas le même sens ni le même aspect dans les deux films, mais qui se retrouve chez le danseur âgé et chez Bill, une fois qu'ils ont été démasqués, comme un symbole de leur vanité ; les guirlandes lumineuses, omniprésentes dans le film de Kubrick, mais aussi dans le décor du Palais de la danse chez Ophüls, qui inscrivent la dimension festive au cour des deux films, la fête étant un moment de dépense éphémère qui a pu aussi acquérir une dimension connotative liée au thème de la vanité.

Lallusion au Plaisir oriente donc la vision d'Eyes Wide Shut en mettant sur la voie du thème de la vanité. Par rapport au film d'Ophüls, Kubrick propose sur ce thème une variation. Dans Le Plaisir, Ophüls mêle trois dimensions propres à la vanité : la recherche masculine du plaisir, éphémère, dépensière d'énergie, par opposition au bonheur conjugal ; l'attention portée à l'apparence, thème qu'on trouve d'ailleurs dans d'autres nouvelles de Maupassant (La Parure $)^{13}$; la vanité métaphysique de la lutte contre le vieillissement et la mort. Dans Eyes Wide Shut, la recherche masculine du plaisir est également une forme essentielle de vanité, mais la femme n'est pas en reste, puisqu'elle ne se contente pas du bonheur conjugal ${ }^{14}$. L'attention portée à l'apparence subsiste, mais prend un autre sens du fait que le thème de la vanité métaphysique de la lutte contre le vieillissement et la mort ne concerne que des personnages secondaires (mort d'un des patients de Bill, séropositivité d'une prostituée). Bill est encore jeune, et donc pas encore touché par ce problème. En revanche, il doit prendre soin de son apparence en louant des vêtements pour entrer dans l'orgie, intégrant là un monde auquel il ne devrait pas avoir accès, d'hommes riches et puissants. Plus que la vanité métaphysique, c'est donc la vanité sociale qui est le thème principal d'Eyes Wide Shut, l'envie d'appartenir à une sphère sociale qui semble en même temps inaccessible, à la fois invisible

13 Guy de Maupassant, La Parure (1884), repris dans La Parure et autres scènes de la vie parisienne, Paris, Flammarion, 2006, p. 23-35.

14 La recherche du plaisir n'est pas non plus l'exclusivité des hommes chez Ophüls, comme le montrent certains sketches de La Ronde. 
(à l'exception de Ziegler, Bill ne saura jamais qui sont ces gens), inatteignable et omniprésente (à partir du moment où Bill est repéré, il est suivi et tous ses faits et gestes sont connus). L'échec de sa tentative d'intégrer ce monde est d'ailleurs résumé par la séquence où Bill reçoit un message à travers le portail du manoir où s'est déroulée l'orgie, lui intimant de cesser immédiatement toute recherche sur ce qu'il a vu, tandis qu'une caméra située plus haut que lui le filme à côté d'un globe, symbole de toute-puissance divine ${ }^{15}$. Mais en liant cette aventure vécue par Bill au récit du fantasme de sa femme, Kubrick suggère en même temps que cette vanité sociale, qui est une impuissance à accéder à un monde plus élevé, est la traduction dans l'espace public d'une impuissance sexuelle dans la sphère privée. La dernière phrase prononcée par Alice - qui ne se trouve pas dans la nouvelle de Schnitzler - semble le confirmer, rappelant à son mari qu'il est temps de «baiser".

Eyes Wide Shut n'est pas le seul film de Kubrick où celui-ci se penche sur la vanité de la vie humaine. Son versant social est également évoqué dans Barry Lyndon (1975), sa dimension métaphysique, liée au passage du temps, dans Lolita (1962). 2001, L'Odyssée de l'espace (1968) aborde aussi la vanité métaphysique sous l'angle de la quête de connaissance de l'Humanité, à jamais éperdue et inachevée. Les entreprises humaines chez Kubrick sont souvent vaines, condamnées à l'échec. Collectivement, quand on s'entraîne pour la guerre en pensant que ça suffira pour la gagner (Full Metal Jacket, 1987), ou qu'on imagine des programmes de réinsertion des délinquants (Orange mécanique, 1971). Mais aussi individuellement, comme Jack Torrance, incapable d'écrire son roman et sombrant dans la folie, dans Shining (1980). On cherche parfois ce qui permet de lier thématiquement les films de Kubrick, dont on connaît le caractère hétérogène sur le plan de leur affiliation générique. La bifurcation intertextuelle, ouvrant sur une allusion au Plaisir d'Ophüls, permet de reconnaître en la vanité l'un de ces thèmes. Elle est l'une des caractéristiques fondamentales, pour Kubrick, de l'existence humaine.

Emmanuel Plasseraud

MCF en études cinématographiques et audiovisuelles Université Bordeaux Montaigne

Équipe CLARE - Centre ARTES emmanuel.plasseraud@u-bordeaux-montaigne.fr

15 Voir Peter Sloterdijk, Globes, Sphères II, Paris, Méta-Éditions, 2010. 


\title{
Résumé
}

Adaptation de La Nouvelle Rêvée d'Arthur Schnitzler, Eyes Wide Shut propose une bifurcation intertextuelle au cours de laquelle le film intègre au récit, qui dans l'ensemble suit plutôt fidèlement celui de la nouvelle, une référence au premier sketch du Plaisir de Max Ophüls. Cette référence est l'occasion d'une étude sur les jeux de renvois qu'opère Kubrick entre Schnitzler, Maupassant et Ophüls, qui oriente l'interprétation que l'on peut donner du film dans le sens d'une réflexion sur la vanité de l'existence, thème qui se trouve chez ces trois auteurs mais que Kubrick prolonge à sa manière.

\section{Mots-clés}

Max Ophüls, intertextualité, Eyes Wide Shut, Maupassant, Schnitzler.

\begin{abstract}
Adapted from Arthur Schnitzler's "Dream Story", "Eyes Wide Shut" offers an intertextual curve during which the film's narrative -which overall follows the novella rather faithfully-integrates a reference to the first sketch of Max Ophüls's "le Plaisir". This reference allows for a study of the cross references between Schnitlzer, Maupassant and Ophüls, which calls for an interpretation of the film centred on the vanity of existence, a theme common to all three authors that Kubrick deals with his own way.
\end{abstract}

\section{Keywords}

Max Ophüls, intertextuality, Eyes Wide Shut, Maupassant, Schnitzler. 


\section{NOTICE BIOGRAPHIQUE}

Emmanuel Plasseraud est maître de conférences en Études cinématographiques à l'Université Bordeaux Montaigne, dont il co-dirige le Master "Documentaire et archives ». Il a publié deux ouvrages aux Presses Universitaires du Septentrion, Cinéma et imaginaire baroque (2007) et L'Art des foules, théories de la réception filmique comme phénomène collectif en France (1908-1930) (2011), ainsi que des articles dans des revues comme Vertigo, Ligeia, Cinergon ou Éclipses, ayant pour sujet Kubrick, mais aussi quelques cinéastes russes (Lopouchanski, Yufit), d'Europe centrale (Skolimowski, Huzsarik), ou encore Raoul Ruiz, Carmelo Bene et Satoshi Kon. Il travaille actuellement à une histoire de la théorisation de la réception filmique comme phénomène collectif et comme expérience individuelle. Il est aussi réalisateur de films de fiction et de documentaires. 